\title{
Lire ou voir la poésie: Le dilemme de la fusion des genres
}

\author{
Amal Abdel-Sattar Abd-Allah Abdel-Karim \\ Faculté d'Al-Alsun, Université de Minia, Égypte \\ Email: amal.abdallah@mu.edu.eg
}

A long history of correlation between painting and poetry led to the arrival of a joint project between R. Queneau and J.-P. Vroom to comment in poetry on surrealist plates made by the latter who shares an almost common vision with the poet.

This is part of a different perspective from that of Apollinaire's calligrams, which marked a revolution in modern visual poetry.

Because, in the case of calligrams, the pictorial presence offers a mono-existence of the imaged text. But in the case of the Monuments, Queneau's collection, the two expressions co-exist reflecting two points of view on a single theme: the image with the poem; the poetic text rests next to the painting: two products of a single data that is the title.

In this study, we try to restore the invisible link between the given title, the image and the poem. The suggested interpretation is, therefore, only our personal view of the entire image; which gives art its potentiality, even its beauty.

Keywords: poetry, painting, visual writing, artistic interpretation

\section{Résumé}

Une longue histoire de corrélation entre peinture et poésie a permis l'arrivée à un projet commun entre R. Queneau et J.-P. Vroom de 
commenter en poésie des planches surréalistes faites par ce dernier qui partage une vision presque commune avec le poète.

Cela s'inscrit dans une perspective différente de celle des calligrammes d'Apollinaire qui marquait une révolution dans la poésie visuelle moderne. Car, dans le cas des calligrammes, la présence picturale offre une mono-existence du texte imagé. Mais dans le cas des Monuments, le recueil de Queneau, les deux expressions co-existent reflétant deux points de vue sur un seul thème: l'image avec le poème; le texte poétique se repose à côté de la peinture: deux produits d'une seule donnée qui soit le titre. Dans cette recherche, on essaie de restaurer le lien invisible entre le titre donné, l'image et le poème. L'interprétation suggérée n'est, par conséquent, que notre vision personnelle de l'mage entière; ce qui donne à l'art sa potentialité, voire sa beauté.

\section{Mots-clés}

Poésie, peinture, écriture visuelle, interprétation artistique.

\section{Introduction}

La poésie dans sa relation avec le vu n'a pas trop loin dépassé l'héritage poétique enlisé dans l'image stéréotypée et les clichés poétiques. Il est à signaler que la corrélation entre la peinture et l'écriture poétique devait avoir ses racines non seulement chez un Apollinaire qui a vu dans ses calligrammes (Beaumarchais, 1994, pp. 248-249) la chance d'écrire en beauté à travers le renvoi d'un sens à l'imagination visuelle, mais aussi, avant lui, cette idée de lier le dessin à la poésie trouve son premier écho chez le poète grec Simmias de Rhodes (IV ${ }^{\text {ème }}$ siècle av. J.-C.), puis chez Rabelais au XVI ${ }^{\text {ème }}$ siècle et à la fin du XIX ${ }^{\text {ème }}$ siècle le genre était pratiqué chez Edmond Haraucourt. Bien qu'audacieuse, cette démarche, qui implique un mariage ambitieux entre le texte poétique et l'image, n'a point osé, la plupart du temps, de révéler plus d'un aspect amusant qui ne dépasse pas le divertissement visuel. Le texte poétique s'est devenu un tableau de valeur artistique variable, dont l'auteur est en même temps poète et dessinateur, s'il est question de calligrammes par exemple; et par conséquent, le rôle du lecteur est devenu celui d'un critique adressé à méditer une production émanant d'une seule source, voire d'un seul 
auteur.

Avec certains poètes modernes et contemporains dont nous avons Queneau comme un modèle, l'expérience poétique rompt avec l'habituel en nouant un nouveau contrat avec le lecteur. Ce contrat permettrait la coexistence de deux auteurs traduisant, chacun à son tour, le texte poétique selon sa propre vision; l'un est un poète, l'autre est un artiste. En cela, la diversité des perspectives et celle des interprétations jaillissent, en donnant l'espace au lecteur d'opter l'un ou l'autre des outils offerts, soient poétiques ou artistiques, et de favoriser l'un ou l'autre tout simplement. À travers cette recherche nous posons la question suivante à laquelle nous essayons de donner une réponse: l'avenir poétique, serait-il en faveur de cette tendance encourageant la fusion des genres artistiques?

\section{Le statut de l'auteur}

Le postulat de l'unicité de l'auteur dans une œuvre poétique ou une œuvre d'art n'est pas négociable. C'est à lui seul que le lecteur se réfère dans l'acte de la réception. La mise en cause de cette tutelle longtemps déléguée à un seul auteur est digne d'être attribuée à une nouvelle autorité où la vision serait plus ample et l'interprétation plus ouverte.

\subsection{L'autorité unique}

Dans la poésie, comme un genre littéraire très ancien aux formes variées, l'importance dominante est accordée à la forme, c'est-à-dire au signifiant. C'est, donc, un art du langage qui fait une utilisation maximale des

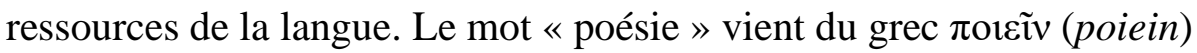
qui signifie «faire, créer». Le nom, poêsis (du latin poesis) signifie "création", et le poète se fait par conséquent un créateur (Larousse, 1997, p. 5802), un inventeur de procédés expressifs et esthétiques, ce que nous trouvons aussi dans les termes du Moyen Âge, comme trouvère et troubadour. Le poète, héritier d'une longue tradition orale, privilégie la musicalité d'où, dans la plupart des textes poétiques, le recours au vers qui apporte aussi la densité. Mais le poète recherche aussi l'expressivité par le poids accordé aux mots comme par l'utilisation fréquente des figures de styles et au premier chef des images, comparaisons et 
métaphores, recherchées pour leur force suggestive. En ce cas, l'écriture s'avère l'outil le plus utilisable de la part du poète qui vise enfin l'image poétique. La notion de l'image n'est plus exclusivement attribuée à la poéticité du texte poétique; elle le dépasse pour représenter la visualité. L'art des calligrammes est l'exemple le plus commun à ce propos. Le calligramme, est un "poème dont les vers sont disposés de façon à former un dessin évoquant le même objet que le texte" (Dictionnaire Le Nouveau Petit Robert, 1993, p. 324) L'auteur, poète ou dessinateur, en ce cas est un.

\subsection{L'autorité plurielle}

La poésie semble s'émanciper de la domination d'un seul auteur.

Raymond Queneau, à la demande de Paul Vroom et en collaboration avec lui, a fait une expérience poétique pareille quand il a écrit Monuments, un recueil de douze poèmes et planches, où le texte se repose en face de l'image. Puisque le poète, avec sa plume, et le sculpteur, avec son pinceau, ont deux visions différentes d'un seul thème poétique.

\section{Le statut du texte poétique par rapport à l'image}

Aucun changement ne peut arriver au texte poétique: il reste le même, soit seul ou en présence de l'image qui se fait parfois par le texte luimême, comme dans les calligrammes, ou par la coexistence avec une image, qui est le tableau traduisant le thème poétique au gré de son artiste; tout par autonomie du texte dont l'interprète est le poète luimême. Ces trois cas de l'existence picturale se montrent ainsi:

\subsection{Degré zéro de la présence picturale}

Dans ce cas, le texte poétique figure seul. L'alignement des vers est celui d'un poème ordinaire. Les poèmes en forme fixe ou en vers libres peuvent représenter le degré zéro de la présence picturale, où l'image poétique existe et l'image visuelle n'a pas de place.

\subsection{Premier degré de la présence picturale}

En dépit de la longue tradition des vers figurés, les calligrammes se distinguent par l'originalité de leur inspiration. Traditionnellement, ce genre était réservé aux poètes religieux ou philosophiques, et ce n'est pas 
par hasard que bon nombre des œuvres primitives prennent la forme d'autels, d'ailes ou d'œufs. (Abd-Allah, 2005, p. 27) Les calligrammes font leurs prolongements dans la fin du XIX e siècle chez un Mallarmé et au début du XX e siècle chez un Apollinaire; l'ensemble de strophes contribue à édifier une structure ou architecture agréable à l'œil. Le poème de Queneau, Morale élémentaire, est de ce type calligrammatique; puisque ses poèmes dans leur ensemble représentent une suite de papillons ou d'arbres (Jouet, 2007, p. 12).

À la suite d'un article intitulé "Framed and shaped writing" paru dans Studio international, en septembre 1968, Peter Mayer, calligraphe et poète concret, parvient à construire une typologie figurative universelle couvrant à la fois la prose et la poésie. Les deux divisions principales sont: «écriture encadrée» et «écriture informée» (Bohn,1981):

A. dans l'écriture encadrée, qui a une plus longue histoire que l'écriture informée, il s'agit d'un mot, ou de plusieurs mots, entourés d'un dessin linéaire. Le message linguistique est enfermé par un trait en forme de dessin.

B. l'écriture informée, au contraire, produit sa propre figuration sans recours à des auxiliaires artistiques. Elle se compose entièrement d'éléments linguistiques et comprend trois sous-catégories:

a. formes compactes, c'est le type le plus commun de la poésie figurée en Occident; ces formes sont remarquables pour leur densité linguistique. Corps de mots compact, le texte est composé d'une suite verticale de vers horizontaux, dont chaque longueur est différente pour produire un contour en forme d'objet sans l'aide d'un trait extérieur. La figure suivante est un poème d'Apollinaire qui représente la tour Eiffel avec des mots séparés, stratifiés horizontalement:

Aussi trouvons-nous un exemple pareil dans le poème suivant d'Apollinaire, qui a subtilement utilisé des vers horizontaux avec d'autres en forme de lignes courbées pour figurer une colombe poignardée et un jet d'eau. Cette forme est un mélange entre ce type et le type suivant. 


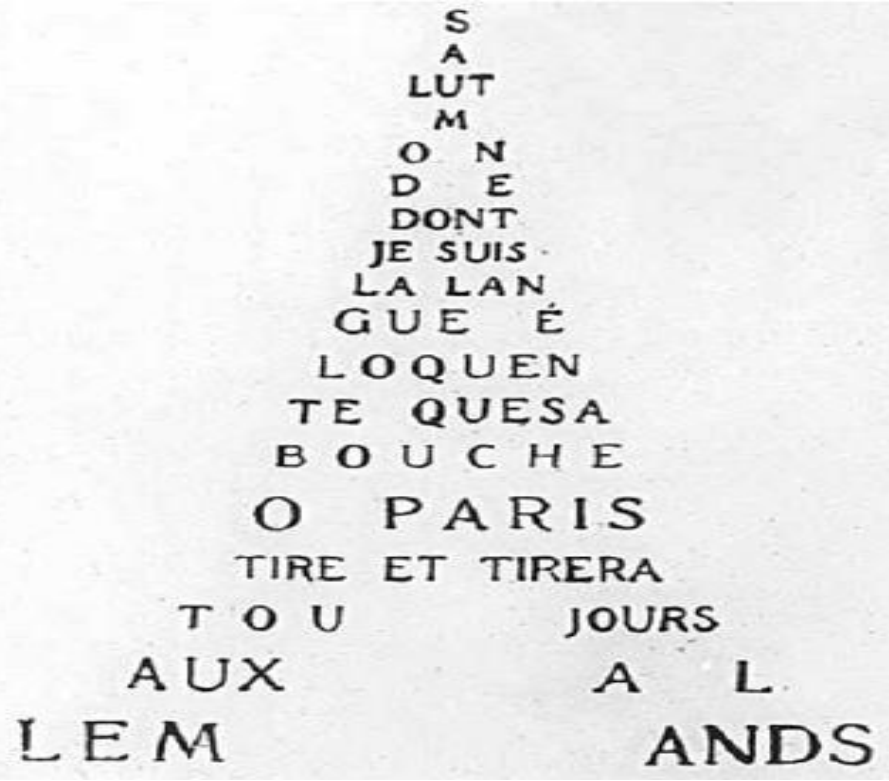

Figure 1: Un poème d'Apollinaire représentant la tour Eiffel

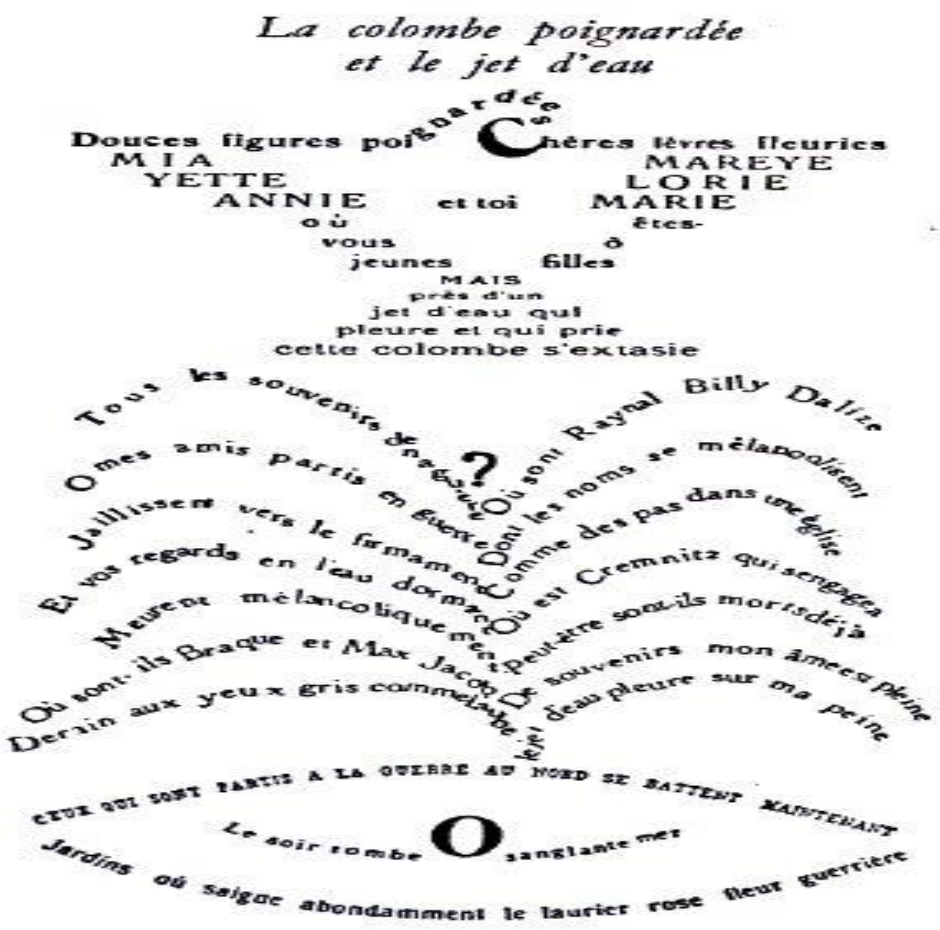

Figure 1: Un poème figurant une colombe poignardée et un jet d'eau 
Un autre poème d'Apollinaire pourrait s'accorder à ce type compact, mais les vers sont en guise de simples lignes verticales irrégulières pour apparaître comme la pluie:

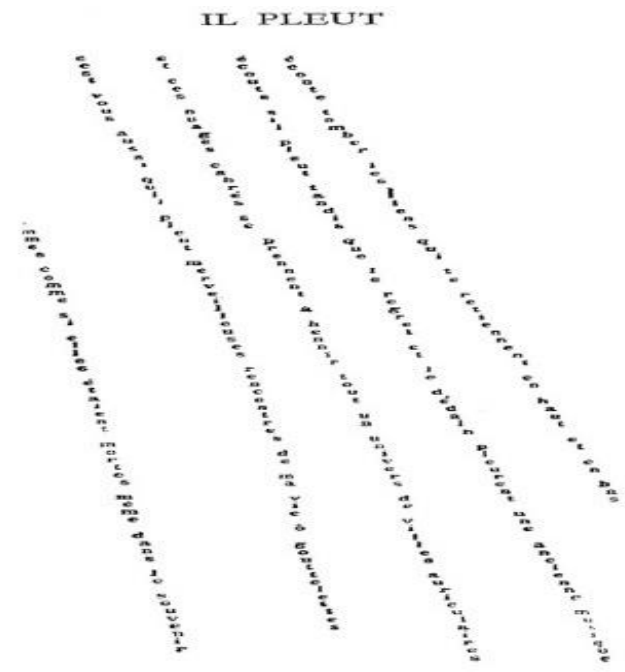

Figure 2: Un poème intitulé Il pleut, montrant des gouttes de pluie

b. formes créées de contours (fermées ou ouvertes), qui sont remarquables pour leur plasticité linguistique. Dans ce cas, le texte est réduit à une ou à plusieurs lignes reproduisant le contour d'un objet donné. Les formes closes tendent à enfermer une grande quantité d'espace mais peuvent avoir quelques mots supplémentaires à l'intérieur. De ce type nous pouvons voir l'image suivante: elle est un peu compliquée que les précédentes. Sa plasticité réside dans la souplesse des lignes utilisées, qui sont une pure écriture manuelle.

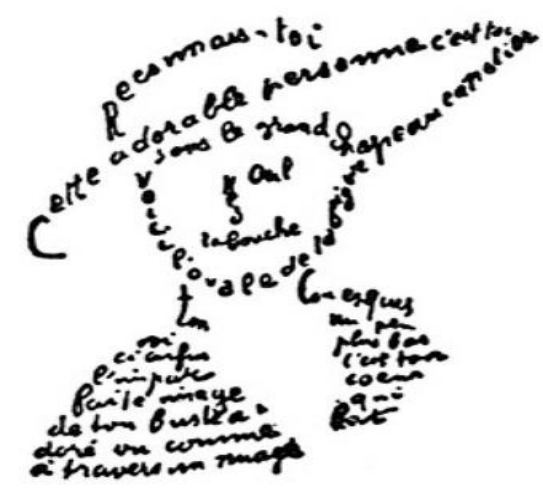

Figure 3: Une figure montrant un poème sous forme d'une femme portant un chapeau 
c. formes à lettres déformées, qui tirent leurs effets du caractère plastique de la lettre. Objet calligraphique par excellence, où la forme de l'œuvre est déterminée par une ou plusieurs lettres déformées de façon picturale. Ce type d'écriture informée est largement répandu dans les pays arabes. On le trouve souvent aussi dans les publicités. (Bohn, 1981)

Ce type de poèmes est commun dans des ouvrages poétiques qui en disposent plus ou moins fréquemment. Apollinaire, dans ses Calligrammes, a offert des types variés qui s'apparentent à l'échelle précédente. Mais, ce type d'écriture poétique où le poète se fait en même temps un peintre pourrait courir le risque de l'illisibilité, du déséquilibre et de la discordance entre le message poétique et l'image qui le présente. Cette œuvre ne dépasse pas parfois le divertissement visuel. Même ce dernier pourrait devenir un pur exercice pictural, où l'écriture se dissipe en faveur d'un encombrement de mots illisibles. Ce qui dérange la lecture et entrave la réflexion; juste comme dans le calligramme de la figure $\mathrm{n}^{\circ} 5$.

Nous mettons l'accent, dans notre recherche, sur le type suivant, où la présence picturale est maximale. Autrement dit, l'image se trouve face à face et en parallèle avec le texte écrit; puisque chaque production, poétique ou artistique, correspondrait à une autonomie expressive et une indépendance interprétative.

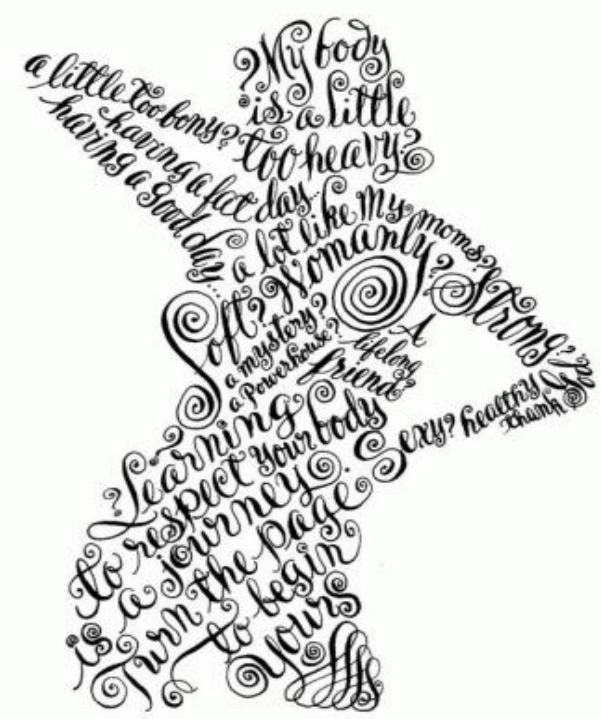

Figure 5: Figure représentant une écriture sous forme d'une silhouette féminine 


\subsection{Deuxième degré de la présence picturale}

Monuments de Queneau est un recueil de poème qui donne le modèle évident de ce type de poésie picturale. Le texte poétique se repose à côté de la peinture: deux produits d'une seule donnée qui soit le titre. Bien que le genre dessin-poème était relativement connu dans la forme des calligrammes, mais le lien entre peinture et poésie ne se restreint pas à ce cadre limité. Cela dépasse encore le poète-peintre qui est la même personne. La vision une et unique se bifurque pour devenir bipède, concernant deux personnes de différentes visions qui essaient, chacune de sa part, de se rencontrer dans un point le plus proche que possible. En conséquence, la critique de l'art contemporain devrait, elle aussi, avoir ses nouvelles dimensions.

Contrairement à la musique, la littérature et la peinture ont en commun d'être deux modes de représentation, deux redoublements de la réalité, et à ce titre leurs rapports sont historiquement à la fois de concurrence et de correspondance. L'époque classique affirmait que la peinture est une poésie muette, la poésie une peinture parlante. (Rajkumar, 2005, p. 45)

Aussi Joanna Rajkumar affirme-t-elle que c'est à partir de XIX ${ }^{\text {ème }}$ siècle que chaque art commence à revendiquer sa spécificité, et que les différences manifestes entre littérature et peinture dont les échanges sont parallèlement rendus possibles se multiplient selon la notion baudelairienne de la correspondance. La peinture s'est affranchie, notamment à l'époque réaliste, de la tutelle littéraire, et elle a cessé en même temps de s'élaborer à partir de sources écrites afin d'affirmer son originalité. Cette indépendance de la peinture s'est accompagnée d'une fascination de la littérature pour les mystères de la création picturale dans laquelle elle a cherché une nouvelle source d'inspiration. (Rajkumar, 2005, pp. 45- 46)

De ce point de vue de la modernité et de l'autonomie de l'art pictural en tant que source d'inspiration littéraire, le lien entre les deux n'est plus celui d'un asservissement ni d'une subordination de la peinture à la littérature, déjà jugée souveraine et dominante. De plus, le projet de Queneau en coopération avec Vroom, même s'il était à la demande de ce dernier, représente un modèle nouveau et flagrant de cette démarche innovatrice de l'inspiration ainsi que du langage poétique moderne dont 
l'image serait exemplaire pour une génération d'artistes et de poètes sur un pied d'égalité.

Mais la question artistique prend d'autres dimensions quand il s'agit d'une existence parallèle, et non pas une coexistence, entre un mode pictural et un poème. La Chine était pionnier dans ce domaine, même si l'on croit que la primauté était occidentale, par les travaux d'un grand peintre et poète chinois, à savoir Wang Wei. Les peintures de celui-ci sont des poèmes et les poèmes sont des peintures. (Martin-Scherrer, 2004, p. 37) L'expérience que nous allons étudier, à ce propos, n'est pas celle d'une simple transposition en poème d'un modèle "pictural" dont l'auteur est un, mais il s'agit de deux auteurs, de deux visions et de deux conceptions parallèles d'une seule idée. Monuments est notre modèle de cette alliance artistique-littéraire.

De ce projet artistique et littéraire compliqué d'un Queneau amateur, philosophe et critique d'art, bien sûr, nous allons étudier quelques poèmes dont l'alliance entre écriture et peinture ne passe pas sans évoquer des significations riches et variées:

Monument pour Giovanni Piranesi, le huitième poème, mémorise l'architecte et graveur italien Giovanni Piranesi, né en 1720 à Mogliano Veneto et mort en 1778 à Rome. Dans ses planches gravées, il parvient à sublimer l'Antiquité. En isolant et en amplifiant les éléments architecturaux, il ajoute à ses œuvres une dimension dramatique qui reflète son idée de la dignité et de la magnificence romaine. Il est l'auteur des eaux-fortes, dont les plus saillantes sont Les prisons (Carceri d'invenzione ou Les prisons imaginaires). C'est une série de seize gravures en deux versions dites deux états, commencée en 1745, montrant d'énormes voûtes souterraines avec des escaliers et de puissantes machines d'une fantastique structure labyrinthique d'un volume épique mais vide de sens explicite. Son art est influencé par le romantisme et le surréalisme. Le poème est le suivant:

Le pire âne est-ce pas çui qui sans dessiner savoir échaffaude l'écrin des prisons un peu mauves 
Çui-ci lui qui savait

transforme les murs ivres

des cordages libèrent

les fils des per-spectives.

(VIII- Monument pour Giovanni Piranesi). (Queneau, 1989, p. 186)

Le jeu de mots ne manque pas à un Queneau tant passionné de ce plaisir: le premier vers est un calembour sur le nom de l'architecte italien Piranesi dont le nom en français est Piranèse ("pire âne est-ce" p.186); cela signifie qu'il s'agit d'un jeu de mots fondé sur la différence de sens entre des mots qui se prononcent de manière identique ou rapprochée. Les vers nous donnent cette idée claire sur un architecte et un graveur doué dont l'œuvre montre la virtuosité évidente d'un dessinateur qui "sans dessiner/savoir/ échaffaude l'écrin des prisons un peu mauves". C'est comme s'il savait déjà créer l'art sans planifier ni préparer, parce que le talent lui est gratuit et spontané. Ses eaux-fortes appelées "Les prisons" sont une pièce d'art, son chef-d'œuvre précieux ressemblant à un "écrin", ou un coffret serrant de chers bijoux. Ces gravures avaient des murs hautains et prestigieux d'inspiration romaine, qui sont d'une hauteur vertigineuse ("Çui-ci [...] transforme les murs ivres"). Ses lignes, comme ses idées, lui sont souples et dociles ressemblant aux cordages et aux fils maniables: ("des cordages libèrent/les fils des perspectives"). Quant à Vroom, c'est le seul portrait humain qui figure parmi ses Monuments. Il s'agit d'un visage d'homme fixant son regard mystérieux, rêveur ou pensif vers le ciel. Son nez est un peu élevé, avec des lèvres serrées et un ensemble de visage désarticulé comme s'il est construit avec de solides pierres.

La partie inférieure de sa joue gauche est vide de pierres, comme si elle tombe ou manque à la construction. Elle est comme un creux obscur et profond d'une petite cave, mais elle donne aussi l'impression dédaléenne très célèbre dans l'art de Piranèse. Ce qui manque au visage du portrait reflète aussi la perspective des mots disloqués n'ayant pas de lien logique entre eux, ni dans les vers eux-mêmes. Un arbre avec de fines branches pousse à côté du visage qui rappelle la dignité et l'orgueil marquant ses dessins et architectures. L'arbre fournit à la gravure ce sens de vie et de beauté qui se mêle à la stabilité et à la puissance de la construction 
lithique.

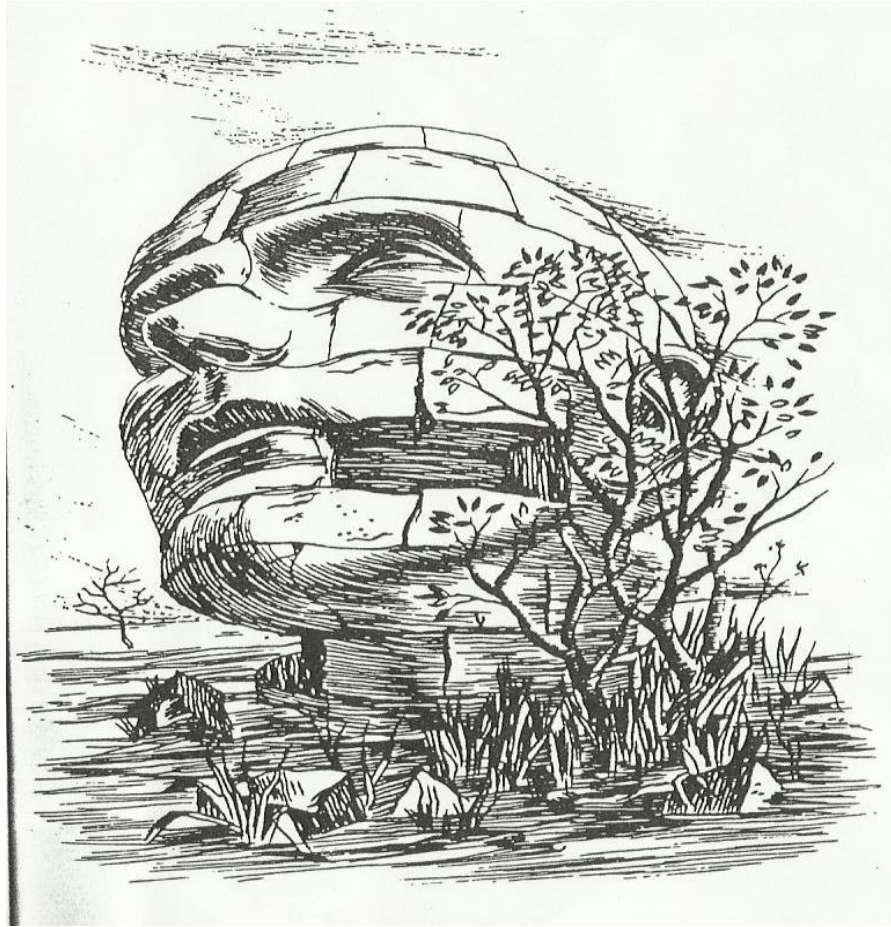

Figure 6: VIII. Monument pour Giovanni Piranesi

Monument aux vivants est un autre poème à contempler: l'attitude étrange de faire une sorte de commémoration aux vivants pourrait se justifier par la volonté de faire d'eux-mêmes, comme vivants, les tuteurs de tout honneur humain, puisqu'ils vivent pour honorer les morts, et les morts survivent grâce à eux. Leur vie est une grâce divine, et s'ils se glorifient, ils le fassent pour toute l'humanité. Monument aux vivants, le deuxième des poèmes artistiques, avec des vers inachevés renvoyant aux corps tronqués du dessin, illustre cet état d'inachèvement que vivent les êtres humains:

Ils ne sont pas achevés

La crême de l'existence

la peau des meilleures nouilles

les santons en moutarde

les croûtons florissants

Ils s'achèvent

et nous, diserts des traits, nous découvrons,

gonflés, vainqueurs des lieux et des communs, 
de ce château, nous diserts, la vie de madrépore. (Monument aux vivants, n. II) (Queneau, 1989, p. 174).

Nous observons que les vers ne sont pas complets. Le "ils" du premier vers correspond à la suite des vers qui vient après: ces sortes de plaisir et de délice rappelant l'ambiance des fêtes et des cérémonies religieuses ne s'achèvent plus. "La crême de l'existence" est en fait une fusion entre le mot carême et crème. C'est une période d'un long jeûne rompu par une fête où la crème de la vie ne se mange pas. Tous les autres aspects cérémoniaux, comme les nouilles, les santons et les croûtons florissants, ne sont que faux parce que non-achevés. La disposition typographique de Queneau lui tient au cœur; il n'oublie pas de parsemer ça et là quelques signes typographiques comme l'espace fait entre les première et deuxième strophes, interposées par un vers isolé "Ils s'achèvent". Mais, qu'est-ce qui se passe quand ces aspects s'achèvent? Nous, les vivants, vainqueurs et gonflés de la gloire victorieuse, découvrons enfin de compte un type de vie différente de celle que nous avons vécu au passé: la vie de madrépore!

En cherchant le lien entre la vie de madrépore et la découverte de cette vie dans un château quelconque, l'image semble compliquée. Le château serait donc celui du bal où les choses n'étaient pas encore complètes. Le mot figure de même dans le poème disloqué, divisé en deux syllabes dont la deuxième est isolée et rejeté à la fin du poème: "ma-drépore". Le madrépore, ou le corail, vit souvent en colonies! Aurait-il une relation avec le concept de la "société"? Aurait-il une correspondance implicite avec la vie sous-marine? Serait-il une allusion à l'au-delà, où on trouve le repos et l'achèvement de toute chose? En ce cas, le titre serait plutôt convenable quand on le change à Monument aux morts et non pas "aux vivants" comme on l'a déjà imposé.

Selon la vision surréaliste des choses et des êtres, les images seraient plus claires quand elles sont associées à leurs contraires; c'est pour cette raison que les deux strophes du poème sont précédées chacune d'un vers contradictoire dans le sens à l'autre: "Ils ne sont pas achevés", "Ils s'achèvent". Le premier vers, à la voix passive, montre la forme verbale présentant l'action comme subie par le sujet. L'action dans le deuxième 
vers est accomplie par le sujet lui-même: le poète se contente de dire "ils s'achèvent" et non pas "ils sont achevés". Donc, la négation du premier vers ne convient pas à l'affirmation du deuxième vers; et par la suite la volonté de faire dans la deuxième strophe n'existe pas dans la première. Les choses qui ont besoin de l'intervention humaine pour s'achever dans la première strophe ne s'achèvent jamais. Les mêmes choses s'achèvent d'elles-mêmes dans la deuxième strophe sans aucune aide de l'homme qui était inactif dans sa vie, et n'existe pas encore dans le château où la fête et la joie. Les vivants dont parle le poème et auxquels le graveur consacre son monument ne sont effectivement, à ses yeux, que des corps amputés gisant dans un château ruiné, que ce soit leur vie inutile et absurde, ou des rêves inachevés ou des actes manqués.

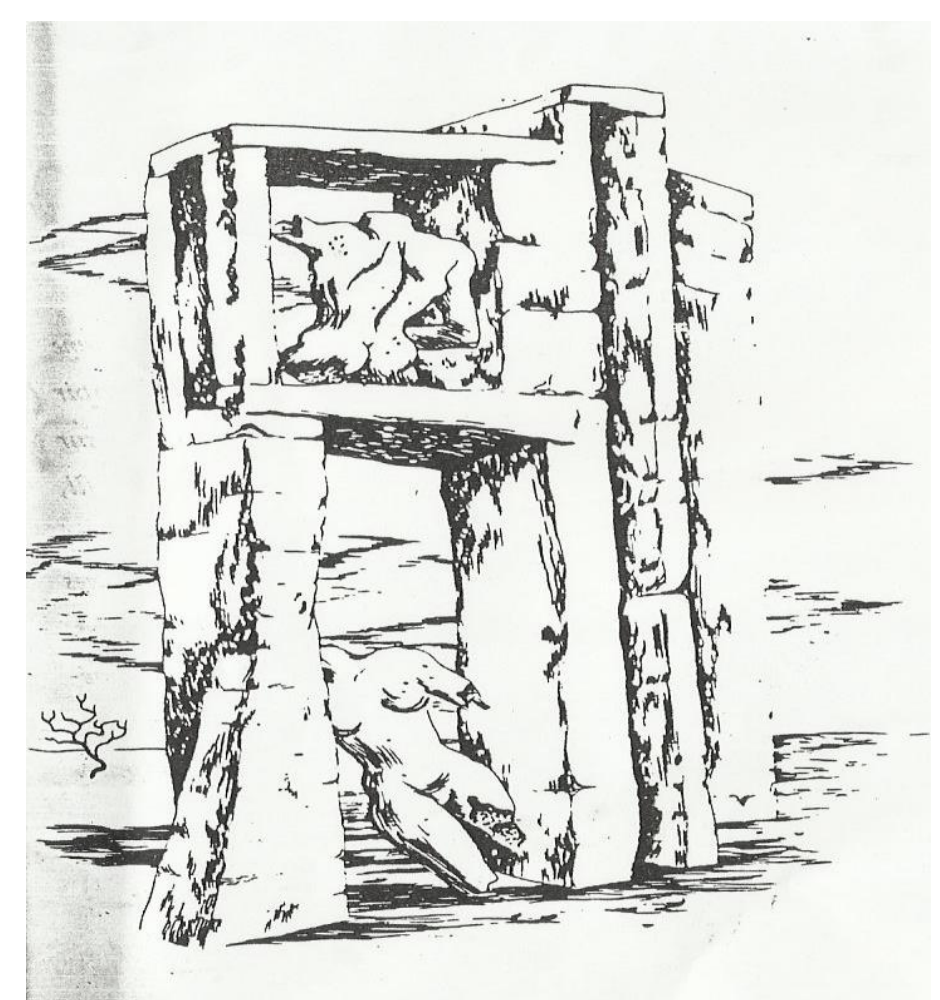

Figure 7: II. Monument aux vivants

Le cinquième poème de Monuments est consacré à commémorer un Monument pour une femme adultère! Le sujet choque pour le sens péjoratif et même insolite que suscite une telle connotation. Mais le 
graveur voulait que l'étrange de titre renvoie plutôt à la virtuosité poétique ainsi qu'à la souplesse éthique plus capables d'aborder des sujets obscènes. Le mot "adultère" paraît plus théologique que l'écriture poétique s'avère de plus en plus spécifique, et le poète, par conséquent s'incombe d'une tâche difficile. Voyons donc ce que Queneau écrit comme commentaire à cette gravure:

Une main sur ses seins

Et l'autre entre ses cuisses

Point ne jette la pierre

Approbateur du Chris.

(V. Monument pour une femme adultère). (Queneau, 1989, p. 180)

La manière dont le poète a composé son poème témoigne de cet état de désordre qu'a vécu cette femme-là au moment de la découverte de son affaire. Les vers chancellent çà et là comme les deux bouts de la balançoire. Le vers avant dernier est une allusion à la parole de JésusChrist aux Pharisiens qui s'apprêtaient à lapider une femme surprise en adultère; que ce soit: "Que celui d'entre vous qui est sans péché lui jette la première pierre" (Debon, 1989). Donc, la plume du poète, à travers la structure même des vers, s'empare le rôle du burin du graveur, et les seuls quatre vers commentant le dessin nous ont donné le sentiment de surprise, de perturbation et de honte de la femme de l'histoire biblique. Pour le dessin, un nouveau désordre s'ajoute à celui fait par le poète. Des choses sont réunies ensemble sans aucun lien logique entre elles: une sorte de paravent du bois, une main droite cachée derrière le paravent en tenant une aiguille à coudre par laquelle passe le fil bien fixé dans le chas, et l'aiguille perce ce qui semble comme une bobine de fil!

En tant que lecteur, la tâche de lire puis de chercher des liens semble de plus en plus difficile, en particulier avec un poète comme Queneau. Mais celle-là s'avérerait impossible quand il s'agit d'une critique d'art. La vue, comme le seul critère de jugement ne suffirait pas pour former cette vision d'ensemble sur des choses plus matérielles. Le critique de l'art visuel, comme le dessin en ce cas, cède à la vision propre de l'artiste; puisque la lecture poétique donne un peu d'espace à l'imagination du lecteur, ce qui ne se réalise pas dans l'art visuel. De plus, une œuvre d'art ne doit pas s'éloigner du vécu. Et c'est bien ce que Vroom essayait de prouver au moyen de sa gravure: les choses ordinaires du dessin, qui 
semblent illogiques dans leur relation ainsi avec le titre qu'avec le poème de Queneau, se montrent aussi comme un rêve. Ce que nous voyons aux rêves n'a aucun lien avec le réel, tandis qu'il existe encore dans le refoulé, c'est-à-dire dans nos inconscients. C'est donc le non-dit dans la réalité, mais qui sera transféré à des symboles dont nous avons seuls les clés. ${ }^{1}$

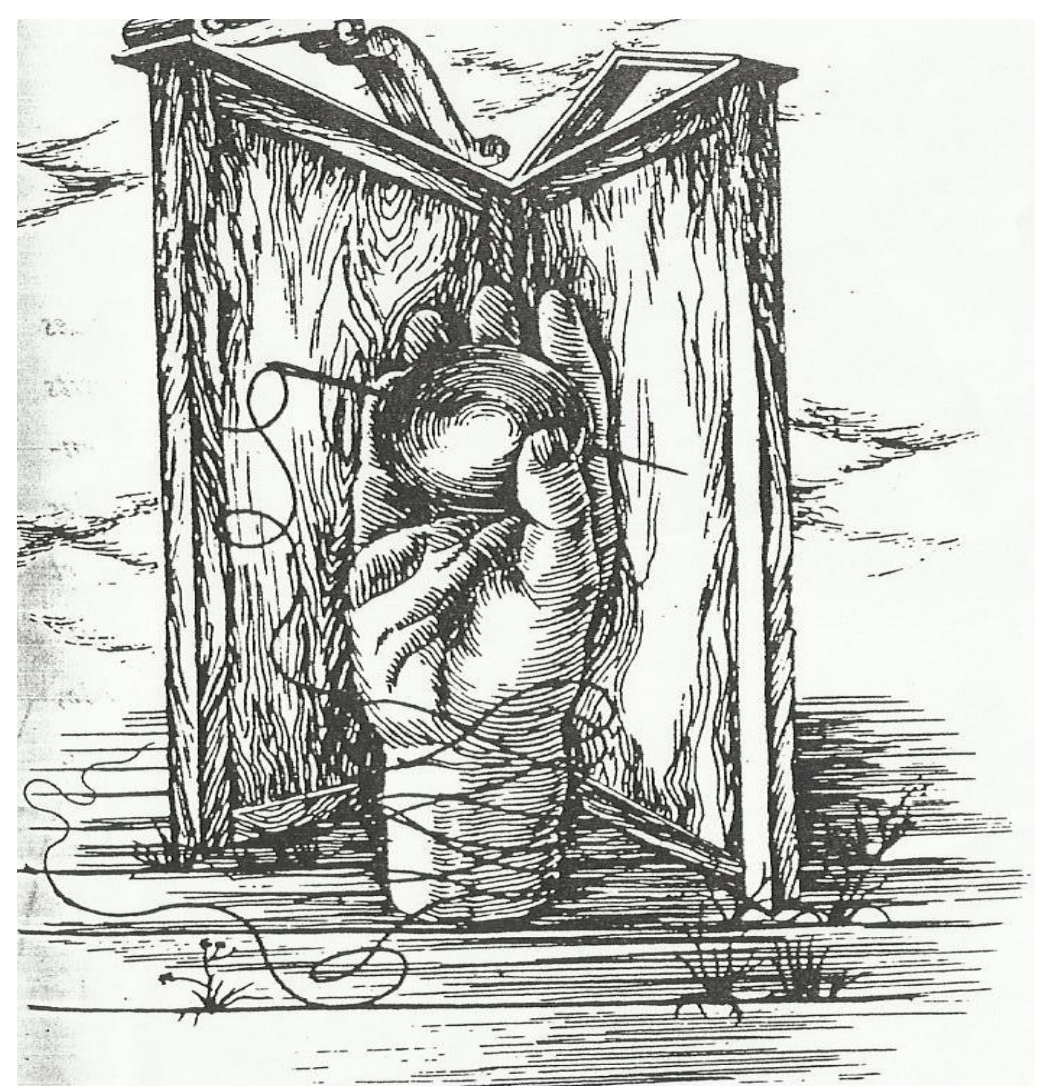

Figure 8: V. Monument pour une femme adultère

En cela, une petite approche pourrait donner des clés pour décrypter le rêve, ou le dessin-rêve de Vroom:

La bobine à la main droite ressemble à la pierre que tiennent les Pharisiens pour lapider la femme adultère. Cette pierre, devenue une

\footnotetext{
1 Dans la théorie psychanalytique freudienne, "interprétation des rêves est la voie royale qui mène à l'inconscient", le rêve possède un sens; il est l'accomplissement d'un désir ayant pour fonction de satisfaire le rêveur.
} 
bobine de fil grâce au désir refoulé du dessinateur, montre cette volonté de ne pas juger l'autrui; et c'est ce que renforce le mot "approbateur" dans le poème. L'aiguille à coudre est cette tentative de recouvrir la nudité d'une femme diffamée, de sauver l'honneur d'une humanité déshonorée et de racheter l'âme d'un être vivant condamné à mort, ainsi que des âmes des gens se faisant en même temps juges et bourreaux. Le paravent serait ce refuge, cet abri protégeant des yeux impitoyables voyant la faute d'autrui en étant aveugles de leurs propres fautes. Cette main droite doit se tendre pour coudre ce qui doit couvrir le corps et le protéger contre les yeux assoiffés à la vengeance, et non pas renverser le paravent ni jeter la première pierre.

Le mythe est aussi un thème poétique d'excellence. Le sixième poème, intitulé Monument pour un chef d'état, pourrait donner une image contemporaine du tyran représenté dans la fausse gloire d'un chef d'état:

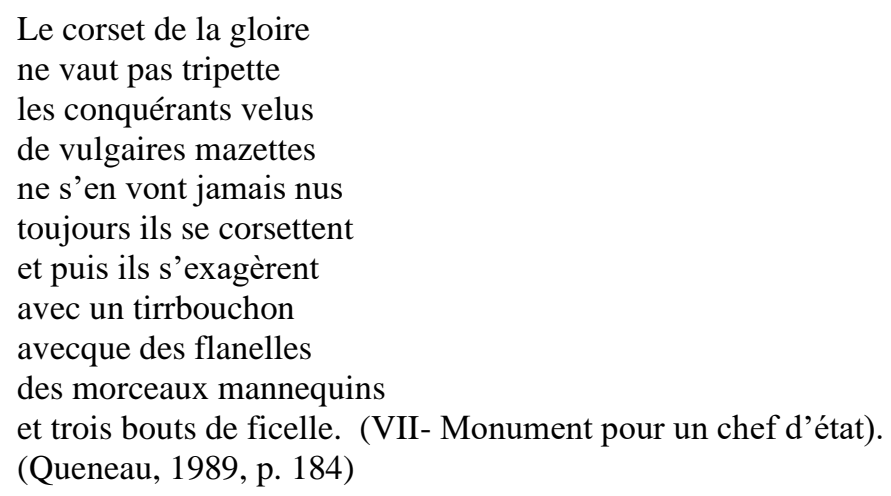

Le cheval et la fausse gloire sont paradoxalement liés: l'image mythique du cheval de Troie ne passe pas sans entraîner une série de dénotations intéressantes. L'épisode du cheval de Troie s'enracine dans la mythologie grecque y voyant le symbole de la ruse: pour la prise de la ville de Troie les Grecs ont eu l'idée de construire un cheval géant en bois creux, dans lequel se cachent des soldats. Convaincus d'accepter l'offrande, les Troyens ont amené le cheval dans l'enceinte de la ville en fête. La torpeur du vin a pris les habitants violemment massacrés par les soldats qui sont sortis du cheval pendant la nuit. Le chevalier, comme Achille, se retire vainqueur de la guerre. Mais un coup de flèche lancé au talon lui coûtait une blessure profonde dont les suites l'ont mené à la mort. (Le 
Larousse élémentaire “pour l'annexe littéraire”, 1956, p. 864 ).

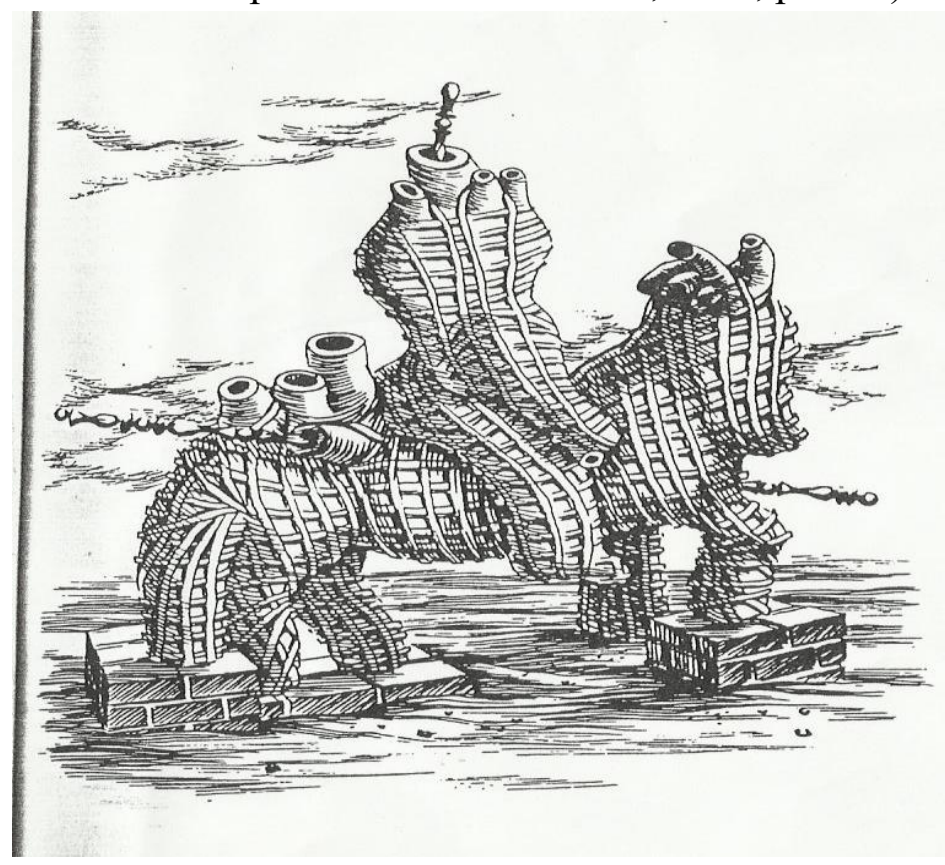

Figure 9: VII. Monument pour un chef d'état

Donc, la gloire ne vaut rien que la mort. Le poète voit que la course des leaders et des guerriers, en tant que chefs d'états, envers la gloire ne vaut rien. Eux, comme des conquérants, ne s'équipent que par des choses banales ("de vulgaires mazettes", "un tirebouchon", "des flanelles" et "trois bouts de ficelle"!), mais toujours ils se renforcent avec le corset de la gloire. Donc, ils sont faux, aussi comme leur fausse gloire.

L'emploi des mots est perturbant; un seul mot donnant un sens pourrait donner un autre sens en même temps: le mot "mazette" signifie un mauvais petit cheval, ce dont on se procure sûrement dans une bataille ou une guerre, mais cela donne aussi le sens d'une personne maladroite et sans aucune habileté, et ce serait le cas pour ce type des gens cherchant la gloire qu'ils ne méritent pas. Le "tirebouchon", même ridiculisé dans le poème par la suppression du "e" muet remplacé par un "r" redoublant le "r" premier, un tirrbouchon, ne trouve pas sa place parmi les équipements nécessaires à une guerre! Mais pour des conquérants ne cherchant que la gloire et la réputation brillante, c'est un outil formé 
d'une hélice nécessaire à enfoncer en tournant dans le bouchon d'une bouteille pour le tirer: la mousse de la champagne qui pétille et s'évapore ensuite est fausse et temporaire comme les faux moments glorieux d'un chef d'état, voire d'un tyran. Celui-ci devient une "marionnette" dans un spectacle: il joue un rôle qui ne lui appartient pas. Il pense ne jouer que le jeu de la politique, mais il se trouve le jeu de celle-là. Les embûches des politiciens, les pièges des opposants, les émeutes et les révoltes ne font que créer une ambiance instable pour un règne dictateur. Et ensuite, les ambitions de ce pauvre chef d'état en font une sorte de "mannequin": il est ainsi ce type imitant ce qu'il n'est pas en fait, il est aussi ce fantoche, cette personne sans caractère qu'on mène aisément comme on veut, il est de même ce modèle marchant avec orgueil et fierté comme on le fait dans un défilé, et enfin il est cette statue, dénudée de vie et servant de modèle pour les peintres et sculpteurs, exactement comme c'est le cas pour la gravure de Vroom! Les trois bouts de ficelle pourraient être celles auxquelles s'attache une marionnette. Mais l'image de cette idole ne restera jamais. Elle s'écroule comme un Jouet mécanique d'enfant. Elle tombe en panne et on trouve que les parties de ce Jouet se désarticulent l'une après l'autre jusqu'à devenir sans valeur. Le chef d'état de Vroom est un Jouet en métal, mais détraqué, abîmé, avec des filaments métalliques sortant de plusieurs endroits de son armature. Le chef décapité, gît paisiblement sur son cheval "mazette", mutilé lui aussi.

\section{Le statut du lecteur}

Par l'extrait suivant de l'ouvrage du philosophe allemand Lessing, nous avons à nous situer, comme lecteurs, dans l'une ou l'autre des trois catégories indiquées par l'auteur.

La première personne qui a comparé la peinture à la poésie était un homme de sentiment, qui savait peut-être que les deux ont produit le même effet sur lui-même. Il s'est senti qu'à travers les deux ce qui est absent semble comme s'il est présent, et l'apparence prend la forme de la réalité [...]. Un autre observateur a exigé de pénétrer sous la surface de ce plaisir, et a découvert que dans la peinture et la poésie, cela s'écoule de la même source; pour la beauté, l'idée de ce que nous soustrairons aux objets physiques, possède les lois générales, applicables à plus d'une classe de choses, aux actions et aux pensées aussi qu'aux formes. Un troisième a réfléchi sur la valeur et la distribution de ces lois générales; et a découvert que 
quelques-unes sont de plus grande force quand on les applique à la peinture, les autres sont ainsi quand on les applique à la poésie [...]. Le premier était l'amateur, le deuxième le philosophe, et le troisième le critique. (Lessing, 1802, pp. xiii-xiv) ${ }^{1}$

En cela, la vision du lecteur d'une œuvre d'art s'inscrirait en tant que vision d'amateur, de philosophe, ou celle d'un critique. Or, la fameuse phrase latine d'Horace Ut pictura poesis (comme la peinture ainsi est la poésie), citée dans son ouvrage Ars Poetica (Art poétique), a tracé le chemin devant une génération de peintres ayant la volonté d'élever la peinture au rang de l'art libéral ${ }^{2}$ en la comparant à l'art honoré de la poésie. (O'donoghue, 2005, pp. 72-80). Le rôle du lecteur, à propos du libéralisme déjà évoqué, ne pourrait point s'échapper à l'une ou l'autre classification faite par Lessing: amateur, philosophe ou critique d'art, tous trois nuancent leur interprétation sur l'échelle de l'ensemble des données poétique et artistique disponibles dans le texte et l'image, tout à fait indépendant l'un de l'autre.

\section{Conclusion}

De tout ce qui précède, nous voyons que la poésie fait ses pas mesurés sur le chemin de la liberté de l'expression poétique. Bien qu'il n'y ait pas de démarche sérieuse vers la libération complète de la tutelle littéraire sur la poésie, mais les pas déjà franchis à ce propos, depuis Mallarmé et Apollinaire en passant par Queneau, sont des prémices d'un avenir poétique audacieux. La démarche déjà prise de Queneau n'est pas habituelle dans la poésie: il a laissé l'espace devant la pratique inconditionnée de l'art, même en conservant son espace de poète, sans que l'un nuise à l'autre. Le futur de la poésie serait dorénavant celui d'un

\footnotetext{
1 A propos du titre de l'ouvrage de Lessing, Laocoon: essai sur les limites entre peinture et poésie, Laocoon est une sculpture romaine qui représente le prêtre Laocoon en lutte avec de grands serpents se lovant autour de lui en essayant de le tuer par leur poison venimeux. Virgile a décrit l'action en vers; Lessing, le philosophe allemand a écrit son essai en 1766 sur la comparaison entre ces deux impressions artistiques en bouleversant tout héritage classique ne voyant aucune différence entre poésie et peinture en tant que deux expressions artistiques étroitement liées.

${ }^{2}$ Le mot "libéral" renvoie à un article publié dans l'URJ (Undergraduate Research Journal). Voir Shannon O’Donoghue supra. Le "libéralisme" mentionné marque la séparation entre poésie et peinture, puisque la deuxième, comme la sculpture, n'est qu'une sorte d'expression esthétique débarrassée des contraintes imposées à la poésie. C'est plus tolérant et large en face du sacré qu'est le mot.
} 
procédé de production et de consommation réciproques: la poésie ne pourrait se restreindre à un seul producteur, puisque de différentes visions peuvent participer à créer soit le texte ou l'image; le texte poétique pourrait se situer, au gré de son, ou de ses auteurs, puisque chacun lui donnerait sa propre vision sans être influencé de l'autre; enfin, le lecteur prendrait sa part à interpréter le texte/image et restaurer son réseau d'interprétations, de comparaisons et de prédilection bien accordée à l'un ou à l'autre procédé utilisé, soit poétique ou artistique.

\section{Bibliographie}

Abd-Allah, A. A.-S. (2005). Tradition et potentialité dans l'œuvre poétique de Raymond Queneau. (Thèse de doctorat non publiée). Université Minia, Minia.

Beaumarchais, J.-P. d. (1994). Dictionnaire des œuvres littéraires de langue française (A-C). In J.-P. d. Beaumarchais, Dictionnaire des œuvres littéraires de langue française (A-C) (pp. 248-249.). Bordas.

Bohn, W. (1981). L'imagination plastique des calligrammes in lecture et interprétation des calligrammes. Actes du Colloque de Stavelot (1977). Bulletin de l'Association Internationale des Amis de Guillaume Apollinaire Jambes, 9(2930), 1-23.

Debon, C. (1989). Notes et variantes in Euvres complètes de Raymond Queneau. In R. Queneau, Euvres complètes de Raymond Queneau (p.1233). Gallimard.

Dictionnaire Le Nouveau Petit Robert. (1993). Dictionnaire Le Nouveau Petit Robert. In Dictionnaire Le Nouveau Petit Robert (p. 324). Edition le Robert.

Jouet, J. (2007). La morale élémentaire. (P. U. Rennes, Ed.) La Licorne.

Larousse, L. G. U. (1997). Grand Usuel Larousse (Dict. encyclopédique), vol. IV (p. 5802). Larousse-Bordas.

Le Larousse élémentaire "pour l'annexe littéraire". (1956). Librairie Larousse.

Lessing, G. E. (1802). Du Laocoon, ou, des limites respectives de la poésie et de la peinture. Chez Antoine-Augustin Renouard.

Martin-Scherrer, F. (2004). Lire la peinture, voir la poésie. In F. Martin-Scherrer, Lire la peinture, voir la poésie (p. 37.). Institut Mémoires de l'Édition Contemporaine.

O'donoghue, S. (2005). Ut Pictura Poesis and the Relationship between Poetry and Painting during the Renaissance. URJ (Undergraduate Research Journal) (Vol. 1), Récupéré de Vol.1, pp. 72-80.

Queneau, R. (1989). Monuments in Euvres complètes. Gallimard (nrf).

Rajkumar, J. (2005, Septembre). Désir de langage et aventure de lignes: Littérature et peinture chez Baudelaire, Hofmannstahl et Michaux. La Revue Rilune (Revue des littératures de L'Union Européenne de l'université de Bologne). Récupéré de http://www.rilune.org/mono2/posie01.htm 